\title{
Pathways from insecure attachment to paranoia: the mediating role of emotion regulation
}

\author{
Olivia Partridge ${ }^{\star}$ (B), Tess Maguire and Katherine Newman-Taylor (i) \\ Department of Psychology, University of Southampton, Southampton, UK \\ ${ }^{*}$ Corresponding author. Email: o.partridge1@gmail.com
}

(Received 30 April 2021; revised 25 October 2021; accepted 9 December 2021; first published online 24 January 2022)

\begin{abstract}
Background: Paranoia is common across the clinical and non-clinical spectrum. Cognitive behavioural therapy for psychosis currently yields modest results, warranting research into symptom-specific maintenance factors to improve outcomes. There is strong evidence of a relationship between insecure attachment and increased paranoia, but little is known about the mediating mechanisms. Emotion dysregulation is associated with both insecure attachment and paranoia, and a candidate causal mechanism. Aims: This study aimed to determine if emotion dysregulation mediates the association between attachment and paranoia.

Method: Sixty-two individuals with elevated paranoia were recruited from NHS services and community settings across the South of England. Mediation analyses were conducted on trait attachment, emotion regulation and paranoia variables, which were collected at one time point.

Results: As predicted, emotion dysregulation mediated the relationship between attachment avoidance and paranoia, and between attachment anxiety and paranoia. Emotion suppression did not mediate the relationship between attachment avoidance and paranoia, possibly due to power. Attachment avoidance correlated with deactivating emotion regulation strategies (e.g. lack of emotional awareness) and attachment anxiety correlated with hyperactivating emotion regulation strategies (e.g. impulse control difficulties). Both deactivating and hyperactivating strategies correlated with paranoia.

Conclusion: Emotion dysregulation is not routinely targeted in cognitive behavioural therapy for psychosis. This study suggests that incorporating emotion regulation strategies in therapy may improve clinical outcomes. Experimental studies are now required to support a causal argument, and pilot intervention studies should investigate if emotion regulation skills development (aligned with attachment style) is effective in reducing non-clinical and clinical paranoia.
\end{abstract}

Keywords: affect regulation; attachment; emotion regulation; mediation; paranoia; psychosis

\section{Introduction}

Paranoia is defined as beliefs of persecution, conspiracy, and threat in the absence of supporting evidence (Freeman, 2007). Paranoia exists in both non-clinical and clinical populations, ranging on a continuum from mild suspiciousness to intense distressing delusions (Freeman et al., 2005; Johns and van Os, 2001). In clinical populations, paranoia is a key symptom of psychosis and is typically associated with schizophrenia-type diagnoses (Cooke, 2014). Clinical paranoia is associated with a range of negative outcomes such as poor treatment response, isolation, emotional distress, and poor quality of life (Freeman, 2016). Given the impact, it is imperative that we understand the factors that lead to its development and maintenance of paranoia, and ensure that these are targeted in psychological interventions.

(C) The Author(s), 2022. Published by Cambridge University Press on behalf of the British Association for Behavioural and Cognitive Psychotherapies. This is an Open Access article, distributed under the terms of the Creative Commons Attribution licence (https:// creativecommons.org/licenses/by/4.0/), which permits unrestricted re-use, distribution, and reproduction in any medium, provided the original work is properly cited. 
Cognitive behavioural therapy (CBT) focuses on cognitive and behavioural change strategies (Morrison and Barratt, 2010) and is currently recommended for individuals experiencing or at risk of psychosis, including symptoms such as paranoia (National Institute for Health and Care Excellence, 2014). Evidence of the effectiveness of CBT for psychosis is inconsistent across meta-analyses. Van der Gaag et al., (2014) found that CBT resulted in modest improvements in hallucinations, although results were less consistent for delusions, and Bighelli et al., (2018) found low to medium improvements across positive symptoms. By contrast, Laws et al., (2018) examined the impact of CBT on functioning in people with psychosis, finding no improvement in quality of life and only a small reduction in distress, which became non-significant when adjusted for possible publication bias. While there is evidence for the effectiveness of CBT, the modest and inconsistent findings suggest we may need to explore additional factors involved in the maintenance of psychosis which are not currently targeted in therapy. One area likely to be therapeutically beneficial is the role of attachment (Berry et al., 2020; Gumley et al., 2014).

\section{Attachment}

Attachment theory assumes that early relationships with primary caregivers influence beliefs about self, others, and the world, as well as emotion regulation, into adulthood (Bowlby, 1969/1982). Attachment style is typically conceptualised along two dimensions: attachment anxiety and attachment avoidance (Brennan et al., 1998). Individuals scoring low on both dimensions are said to have a secure attachment, and tend to hold reasonably positive self and other beliefs and use effective emotion regulation strategies, as a result of responsive caregiving (Mikulincer and Shaver, 2016). Individuals scoring highly on either attachment anxiety or avoidance are said to have an insecure attachment (Brennan et al., 1998). Attachment anxiety typically follows inconsistent caregiving, and is associated with negative representations of the self, and a strong desire for connection coupled with a fear of rejection (Mikulincer and Shaver, 2016). Individuals with high levels of attachment anxiety tend to rely on hyperactivating emotion regulation strategies, such as catastrophising emotion, in an attempt to gain a response from attachment figures (Mikulincer and Shaver, 2016). Attachment avoidance typically follows rejecting caregiving and is associated with negative representations of others and a compulsive self-reliance (Mikulincer and Shaver, 2016). Individuals with high levels of attachment avoidance tend to adopt deactivating emotion regulation strategies, such as emotional suppression, to avoid further rejection from attachment figures (Mikulincer and Shaver, 2016). Both hyperactivating and deactivating strategies function to reduce the emotional pain associated with the unavailability of attachment figures (Mikulincer and Shaver, 2007).

Insecure attachment is associated with increased clinical paranoia, with a recent systematic review finding significant associations in 11 out of the 12 identified studies (Lavin et al., 2020). Insecure attachment is also associated with non-clinical paranoia (Berry et al., 2006; Pickering et al., 2008). Furthermore, a recent meta-analysis found strong associations between both attachment anxiety and avoidance, and paranoia, with no difference between clinical and non-clinical samples (Murphy et al., 2020). Experimental studies have also found that priming insecure attachment increases level of paranoia (Hutton et al., 2017; Sood and Newman-Taylor, 2020).

While there is strong evidence of a relationship between attachment style and paranoia, little is known about the mediating mechanisms underlying this relationship. Read and Gumley (2008) propose that attachment predicts psychosis via a range of mediating mechanisms, including emotion regulation.

\section{Emotion regulation}

Hyperactivating (e.g. catastrophising) and deactivating emotion regulation strategies (e.g. suppression) are typically used by those with insecure attachments (Mikulincer and 
Shaver, 2007; Owens et al., 2013), and are associated with increased psychosis symptomology, including suspiciousness (Dozier and Lee, 1995). This is supported by longitudinal research; for example, suppression predicted increases in paranoia in individuals with psychosis in experience sampling studies (Kimhy et al., 2020; Nittel et al., 2018), and self-blaming predicted non-clinical paranoia one month later (Westermann et al., 2013). In addition, an experimental study found that increases in paranoia after a social exclusion task were accounted for by lower levels of functional (e.g. acceptance) and higher levels of dysfunctional emotion regulation strategies (e.g. catastrophising and blaming others), in individuals at high risk of psychosis (Lincoln et al., 2018). Correlational research shows that dysfunctional emotion regulation (e.g. impulse control difficulties and catastrophising) is associated with paranoia when controlling for a range of variables (Westermann and Lincoln, 2011; Westermann et al., 2013). Importantly, preliminary intervention studies have found that emotion regulation skills training may be an effective way to reduce paranoia in psychosis (Silva et al., 2020).

Limited research has examined emotion regulation as a mediator in the attachment-paranoia relationship in psychosis (Ascone et al., 2020; Castilho et al., 2017; Jones, 2015) and in non-clinical psychotic-type experience (Udachina and Bentall, 2014). There is some evidence that hyperactivating emotion regulation strategies (rumination, self-blame, catastrophising) mediate the relationship between attachment anxiety and clinical paranoia (Ascone et al., 2020), and that internal dysfunctional affect regulation (e.g. dwelling on thoughts and feelings) mediates the relationship between fearful attachment (negative view of self and other) and clinical hallucinations, but not other positive symptoms (Jones, 2015). There is also some evidence that experiential avoidance mediates the relationship between attachment anxiety and nonclinical paranoia (Castilho et al., 2017; Udachina and Bentall, 2014).

Unexpectedly, Jones (2015) found that internal-functional affect regulation (e.g. concentrating on positive activity) mediated the relationship between dismissing (avoidant) attachment and positive symptoms (but not hallucinations). While this strategy is generally considered functional, avoidantly attached individuals tend to supress affect and not seek out others for support; these results may therefore reflect an unhelpful overuse of internal strategies. Experiential avoidance may mediate the relationship between attachment avoidance and non-clinical paranoia (Udachina and Bentall, 2014), although blaming others did not mediate the relationship between attachment avoidance and clinical paranoia (Ascone et al., 2020).

Many of these studies did not control for confounders (Ascone et al., 2020; Castilho et al., 2017; Jones, 2015; Udachina and Bentall, 2014), used weak data analysis (Castilho et al., 2017), and/or employed post-hoc analyses (Ascone et al., 2020), which limit confidence in the results.

The research to date indicates that the recommended psychological interventions for paranoia have just moderate effects, warranting research into other factors that contribute to the maintenance of paranoia. Research suggests that insecure attachment is associated with both emotional dysregulation and paranoia, and that emotion dysregulation is associated with paranoia. We have preliminary evidence that emotion regulation may be involved in the relationship between attachment and psychosis. Robust research examining whether trait emotion regulation mediates the relationship between trait attachment and paranoia would help to isolate key processes which may be amenable to change in therapy. Previous research has also recommended exploring emotional suppression as a mediator in the attachment avoidance and paranoia relationship, as this is assumed to be a key strategy associated with this attachment style (Ascone et al., 2020).

Additionally, it would be beneficial to explore correlations between attachment style and emotion regulation strategies to inform potential hypotheses about tailoring emotion regulation skills training by attachment style for clinical populations. 


\section{Current study}

The current study aims to test the following hypotheses:

H1: Emotion dysregulation [as measured by the Difficulties in Emotion Regulation Scale (DERS); Gratz and Roemer, 2004] will mediate the relationship between attachment anxiety [as measured by the Psychosis Attachment Measure (PAM); Berry et al., 2006], and paranoia [as measured by the Revised Green et al., Paranoid Thoughts Scale (R-GPTS); Freeman et al., 2019]

H2: Emotion dysregulation (as measured by the DERS), will mediate the relationship between attachment avoidance (as measured by the PAM) and paranoia (as measured by the R-GPTS)

H3: Emotional suppression [as measured by the Emotion Regulation Questionnaire (ERQ); Gross and John, 2003], will mediate the relationship between attachment avoidance (as measured by the PAM) and paranoia (as measured by the R-GPTS)

Exploratory analyses will also be conducted to examine associations between attachment style and emotion regulation strategies, to explore the following research questions:

RQ1: Is attachment anxiety (as measured by the PAM) associated with hyperactivating emotion regulation [difficulties engaging in goal-directed behaviour, impulse control difficulties, and limited access to effective emotion regulation strategies (as measured by the DERS)]?

RQ2: Is attachment avoidance (as measured by the PAM), associated with deactivating emotion regulation strategies [non-acceptance of emotions, lack of emotional awareness, and lack of emotional clarity (as measured by the DERS)] and suppression (as measured by the ERQ)?

\section{Method}

\section{Design}

We used a cross-sectional design, collecting data at one time point in line with previous research in this area (e.g. Ascone et al., 2020). Cross-sectional analysis is justified by the theoretical rationale for the temporal ordering of the variables (Fairchild and McDaniel, 2017), as outlined above (i.e. enduring trait attachment predicts current emotion regulation and paranoia, and emotion regulation predicts paranoia). The predictor variable (attachment) is assumed to develop in childhood and remain broadly consistent over time, with the PAM found to be stable in individuals with psychosis (Berry et al., 2008).

\section{Participants}

We recruited individuals with elevated levels of paranoia across a broad spectrum of symptom severity and diagnostic status. Participants were recruited from NHS outpatient settings as well as community settings (e.g. voluntary charities and online support groups for people with psychosis). Recruiting participants from both NHS and community settings is in line with the continuum model of psychosis (Johns and van Os, 2001), and with previous research exploring mediating mechanisms between attachment style and psychosis, and allows for comparison across studies (e.g. Ascone et al., 2020; Pilton et al., 2016; Scott et al., 2020; Wickham et al., 2015).

Inclusion criteria were that participants were over 18 years of age, were fluent in English language (as questionnaires are written and standardised in English), had capacity to give informed consent, and experienced paranoia at the time of the study. Experience of paranoia was assessed using the R-GPTS (Freeman et al., 2019), screening for individuals scoring in at least the elevated range on either subscale (Reference $>9$; Persecution $>4$ ). 


\section{Procedure}

The study protocol was pre-registered in July 2020 on OSF Registries (osf.io/5emjf). Early Intervention for Psychosis services (EIP) and Community Mental Health Teams (CMHT) in the South of England were contacted and provided with the study information. Clinicians were asked to review their caseloads to identify and approach potentially eligible participants for initial consent. The first author then contacted potential participants to discuss the study. The study adhered to NHS guidelines for COVID-19 secure contact (via telephone or face-toface). An online version of the study was advertised to relevant support networks (e.g. National Paranoia Network, Mind Charities, Intervoice) for community participants who accessed the study remotely.

After reading an information sheet and providing informed consent, all participants completed the questionnaires in the following order: R-GPTS, PAM, DERS, ERQ, and were then debriefed. Participants recruited from the community were offered the opportunity to be entered into a draw to win one of three $£ 50$ Amazon vouchers. Participants recruited from the NHS received a reimbursement of $£ 10$ (either cash or voucher) for their time and any incurred travel expenses.

\section{Measures}

Demographic questionnaire

This self-report measure gathered information on age, gender, ethnicity, time since onset of paranoia, and any formal mental health diagnoses received.

\section{Paranoia}

The R-GPTS (Freeman et al., 2019) is a self-report measure of paranoia. The scale consists of 16 items; eight items measure ideas of social reference and ten items measure persecutory ideation. Each item is rated on a 5-point Likert scale from 1 (not at all) to 4 (totally), and scores can be summed to yield two subscales or a total score. Higher scores reflect higher levels of paranoia. The scale has excellent reliability ( $\alpha>.90$; Freeman et al., 2019). Internal consistency for the current sample was also excellent $(\alpha=.91)$.

\section{Attachment}

The PAM (Berry et al., 2006) is a 16-item self-report questionnaire assessing trait attachment anxiety and attachment avoidance. Each item is rated on a 4-point Likert scale from 0 (not at all) to 3 (very much). Each dimension yields a total score, with higher scores reflecting greater attachment anxiety and attachment avoidance. In a population of individuals with psychosis, internal consistency was good for anxiety $(\alpha=.82)$ and acceptable for avoidance $(\alpha=.76$; Berry et al., 2008). Internal consistency for the current sample was good for both attachment anxiety $(\alpha=.81)$ and attachment avoidance $(\alpha=.83)$.

\section{Emotion regulation}

The DERS (Gratz and Roemer, 2004) is a 36-item self-report questionnaire assessing trait emotion regulation strategies. The measure yields a global score and six subscale scores: difficulties engaging in goal directed behaviour when upset, impulse control difficulties, limited access to effective emotion regulation strategies, non-acceptance of emotional responses, lack of emotional awareness, and lack of emotional clarity. We grouped the first three strategies as 'hyperactivating', as these function to increase emotional arousal, and the latter three strategies as 'deactivating', as these function to decrease emotional arousal (cf. Mikulincer et al., 2003). Individuals score items on a 5-point Likert scale from 1 (almost never) to 5 (almost always), with higher scores reflecting greater difficulties with emotion regulation. Internal consistency 
of the DERS is excellent $(\alpha=.93)$, with individual subscales all rated as good or above $(\alpha>.80$; Gratz and Roemer, 2004). In the current sample, internal consistency of the total scale was excellent $(\alpha=.96)$. All individual subscales rated as good or above $(\alpha>.83)$, with the exception of emotional awareness which was acceptable $(\alpha=.71)$.

The ERQ (Gross and John, 2003) is a self-report trait measure of cognitive reappraisal and expressive suppression. We used the 4 -item expressive suppression subscale. Individuals score items on a 7 -point Likert scale ranging from 1 (strongly disagree) to 7 (strongly agree). The expressive suppression scale has acceptable internal consistency $(\alpha=.73$; Gross and John, 2003). In the current sample internal consistency was also acceptable $(\alpha=.77)$.

\section{Statistical analyses}

Microsoft Excel was used to collate data and SPSS 26 for Windows was used for data analysis. There were four occurrences of missing data by four different participants. As there was only one item rating missing for each participant, these were assigned based on the average of scores in the relevant subscale (Tabachnick and Fidell, 2013).

Mediation analyses were conducted using PROCESS (Model 4; Hayes, 2018). Differences in categorical demographic data (gender, recruitment pathway, self-reported diagnosis) on the dependent variable (R-GPTS) were explored using independent $t$-tests/one-way ANOVAs. Following protocols in previous research in the area (Hugill et al., 2017), demographic variables with significant differences in levels of paranoia were controlled for in separate mediation analyses, and compared with the models which did not control for covariates. Exploratory research questions were examined using correlational analyses.

\section{Results}

\section{Sample characteristics}

Seventy-one participants completed the study. A total of 62 participants scored on or above the elevated cut-off on the R-GPTS and were therefore included in the analyses. Of the total, 42 were recruited from community settings, and 20 were recruited from clinical settings. Demographic data for the included participants are presented in Table 1, which also shows that our sample reported levels of paranoia comparable to, or more severe than, previous studies recruiting clinical samples.

We found significant differences in level of paranoia (R-GPTS) by gender $\left(t_{60}=2.05, p=.04\right)$ and recruitment pathway $\left(t_{60}=3.69, p<.001\right)$. Females scored higher than men, and participants recruited from the community scored higher than those recruited from the NHS. We therefore conducted mediation analyses which controlled for gender and recruitment pathway, and compared this with the initial mediation analyses. No significant differences were found in level of paranoia by self-reported diagnosis status $\left(F_{2,59}=3.07, p=.05\right)$. Age and years of suspicious thinking did not correlate with level of paranoia (R-GPTS).

\section{Mediation analyses}

Mediation analyses were conducted to determine if emotion dysregulation mediated the relationship between attachment style and paranoia. Sample size was initially estimated using Kline's (2015) model of 20 participants per parameter, yielding a required sample of 60 participants. Additionally, power for the indirect effect of each model was determined post-hoc using Monte Carlo simulations (10,000 replications and 20,000 draws), as recommended by Schoemann et al., (2017). The R application (https://schoemanna.shinyapps.io/mc_power_med/; Schoemann et al., 2017) revealed that a sample size of 62 was sufficient to achieve the conventional desired power level of .80 (Schoemann et al., 2017) in mediation models 1 and 2. A sample size of 62 yielded .85 power for model 1 and .95 power for model 2. However, model 
Table 1. Demographic data and descriptive statistics $(n=62)$

\begin{tabular}{|c|c|c|}
\hline Variable & Descriptive statistic & Comparison figure \\
\hline \multicolumn{3}{|l|}{ Gender: $n(\%)$} \\
\hline Female & $35(56.5)$ & \\
\hline Male & $27(43.5)$ & \\
\hline Age of participant in years: mean $(S D)$, range & 37.02 (12.71), 18-62 & \\
\hline \multicolumn{3}{|l|}{ Ethnicity: $n(\%)$} \\
\hline White British & $50(80.65)$ & \\
\hline Asian & $4(6.45)$ & \\
\hline White American & $2(3.23)$ & \\
\hline White and Black mixed background & $2(3.23)$ & \\
\hline White other background & $2(3.23)$ & \\
\hline Any other mixed background & $2(3.23)$ & \\
\hline Suspicious thinking in years: mean $(S D)$, range & 15.77 (13.66), $1-50$ & \\
\hline \multicolumn{3}{|l|}{ Self-reported mental health diagnosis: $n(\%)$} \\
\hline Yes & $43(69.35)$ & \\
\hline No & $15(24.19)$ & \\
\hline Not sure & $4(6.45)$ & \\
\hline \multicolumn{3}{|l|}{ Self-reported diagnosis type: $n(\%)$} \\
\hline Depression & $14(20.97)$ & \\
\hline Anxiety & $13(17.74)$ & \\
\hline Schizophrenia & $10(16.13)$ & \\
\hline Emotionally unstable personality disorder & $8(12.90)$ & \\
\hline Psychosis & $5(8.06)$ & \\
\hline Post-traumatic stress disorder & $5(8.06)$ & \\
\hline Bipolar & $3(4.84)$ & \\
\hline Delusional disorder & $1(1.61)$ & \\
\hline Attachment disorder & $1(1.61)$ & \\
\hline Attachment anxiety - PAM: mean (SD) & $14.53(5.61)$ & $10.9(6.5)^{\mathrm{a}}$ \\
\hline Attachment avoidance - PAM: mean (SD) & $14.37(5.59)$ & $15.2(5.2)^{\mathrm{a}}$ \\
\hline Paranoia - Persecution R-GPTS: mean (SD) & $20.66(9.87)$ & $13.7(13.0)^{\mathrm{b}}$ \\
\hline Paranoia - Social Reference R-GPTS: mean (SD) & $18.11(7.67)$ & $15.8(7.42)^{\mathrm{b}}$ \\
\hline Paranoia - Total R-GPTS: mean (SD) & $38.76(15.80)$ & - \\
\hline Emotion dysregulation: DERS, mean (SD) & $110.74(30.74)$ & $101.7(26.8)^{\mathrm{C}}$ \\
\hline Emotion suppression: ERQ, mean (SD) & $4.12(1.48)$ & $4.09(1.42)^{d}$ \\
\hline
\end{tabular}

${ }^{a} 44$ adults with voices recruited from community settings, most had contact with mental health services (Robson and Mason, 2015). ${ }^{b} 1804$ adults with psychotic disorder recruited from clinical settings (Freeman et al., 2019). Score ranges for R-GPTS Persecution: average (0-4), elevated (5-10), moderately severe (11-17), severe (18-27), very severe (28+). Cut-offs for Persecution: $11=$ clinical persecutory ideation, 18 = persecutory delusion. Score ranges for R-GPTS Social Reference: average (0-9), elevated (10-15), moderately severe (1620), severe (21-24), very severe (25+). ${ }^{\circ} 592$ adults with mental health difficulties recruited from clinical settings (Fowler et al., 2016$) .{ }^{d} 44$ adults with schizophrenia recruited from clinical and community settings (Kimhy et al., 2012).

3 was significantly under-powered, with a sample size of 62 yielding .14 power. To achieve .80 power in model 3, a sample size of 825 is required.

Mediation is considered significant if the $95 \%$ confidence interval does not cross the zero value (Hayes, 2018). The percentile bootstrap CI is the recommended method for inferring indirect effects as it balances validity and power considerations (Hayes, 2018).

For model 1, as expected, the total effect of attachment anxiety on paranoia was positive and significant; $c=1.31, S E=.32,95 \%$ CI $[.66,1.95]$. When accounting for the mediator (emotional dysregulation) there was no longer a significant relationship between attachment anxiety and paranoia, $c^{\prime}=.55, S E=.39,95 \%$ CI $[-.22,1.32]$. There was a significant positive relationship between attachment anxiety and emotion dysregulation, $a=3.43, S E=.55,95 \%$ CI [2.32, 4.53], and between emotion dysregulation and paranoia, $b=.22, S E=.07,95 \%$ CI $[.08, .36]$. In line with the hypothesis, the indirect effect was significant, suggesting that emotional dysregulation mediates the relationship between attachment anxiety and paranoia $a b=.76$, $\beta=.27$, bootstrapped $S E=.29$, bootstrapped $95 \%$ CI $[.22,1.37]$; see Fig. 1 . Those scoring higher on attachment anxiety had increased emotional dysregulation, which predicted greater 
Figure 1. Mediation model testing if emotion dysregulation mediates the relationship between attachment anxiety and paranoia. Path coefficients are unstandardised regression coefficients. The value in parentheses is the direct effect $\left(c^{\prime}\right)$ of attachment anxiety on paranoia. ${ }^{\star} p<.05,{ }^{\star \star} p<.01,{ }^{\star \star \star} p<.001$.

Figure 2. Mediation model testing if emotion dysregulation mediates the relationships between attachment avoidance and paranoia. Path coefficients are unstandardised regression coefficients. The value in parentheses is the direct effect $\left(c^{\prime}\right)$ of attachment avoidance on paranoia. ${ }^{\star} p<.05,{ }^{\star \star} p<.01,{ }^{\star \star *} p<.001$.

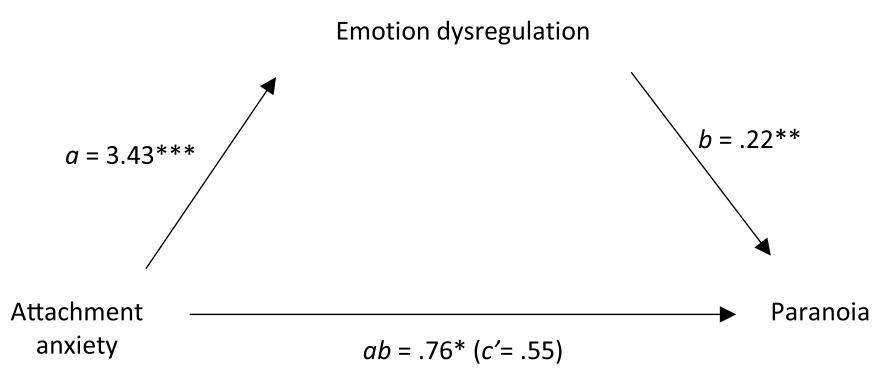

Emotion dysregulation

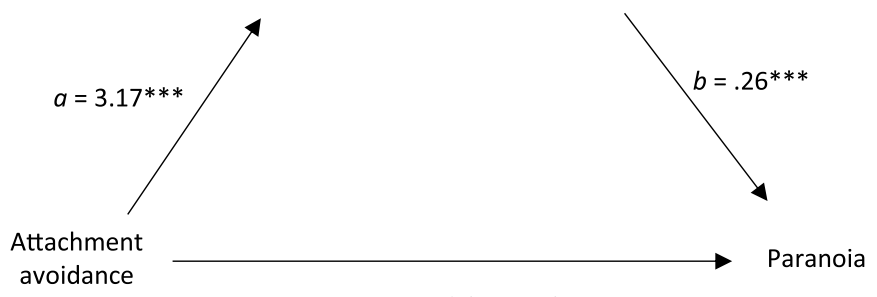

$a b=.83^{*}\left(c^{\prime}=.21\right)$

paranoia. Overall, attachment anxiety and emotional dysregulation explained $33 \%$ of variance in levels of paranoia.

For model 2, as expected, the total effect of attachment avoidance on paranoia was positive and significant, $c=1.04, S E=.34,95 \%$ CI $[.36,1.72]$. When accounting for the mediator (emotional dysregulation) there was no longer a significant relationship between attachment avoidance and paranoia, $c^{\prime}=.21, S E=.37,95 \%$ CI $[-.54, .96]$. There was a significant positive relationship between attachment avoidance and emotion dysregulation, $a=3.17, S E=.58,95 \%$ CI [2.01, 4.33], and between emotion dysregulation and paranoia, $b=.26, S E=.07,95 \%$ CI $[.13, .40]$. In line with the hypothesis, the indirect effect was significant, suggesting that emotional dysregulation mediates the relationship between attachment anxiety and paranoia, $a b=.83, \beta=.29$, bootstrapped $S E=.26$, bootstrapped 95\% CI [.35, 1.39]; see Fig. 2. Those scoring higher on attachment avoidance had increased emotional dysregulation, which predicted more paranoia. Overall, attachment avoidance and emotional dysregulation explained $31 \%$ of variance in levels of paranoia.

For model 3, the total effect of attachment avoidance on paranoia was positive and significant, $c=1.04, S E=.34,95 \%$ CI $[.36,1.72]$. When accounting for the mediator (emotional suppression) there was no longer a significant relationship between attachment avoidance and paranoia, $c^{\prime}=.80, S E=.47,95 \% \mathrm{CI},[-.14,1.74]$. There was a significant positive relationship between attachment avoidance and emotional suppression, $a=.18, S E=.02,95 \%$ CI $[.13, .23]$. There was no significant relationship between emotional suppression and paranoia, $b=1.30$, $S E=1.77,95 \%$ CI $[-2.24,4.84]$. Contrary to prediction, the indirect effect was not significant, suggesting that emotional suppression does not mediate the relationship between attachment avoidance and paranoia, $a b=.24, \beta=.08$, bootstrapped $S E=.34$, bootstrapped 95\% CI [-.41, .96]; see Fig. 3. Overall, attachment avoidance and emotional suppression explained 14\% of variance in levels of paranoia.

Significant mediation models were re-tested controlling for the demographic variables found to exert effects on levels of paranoia (gender and recruitment pathway). Results differed minimally from those already reported. Indirect effects on paranoia remained significant for attachment anxiety $a b=.66, \beta=.23$, bootstrapped $S E=.24$, bootstrapped 95\% CI [.22, 1.15] and attachment avoidance $a b=.62, \beta=.22$, bootstrapped $S E=.23$, bootstrapped 95\% CI [.19, 1.13]. 
Table 2. Correlation matrix for attachment anxiety and emotion regulation (Pearson's $r$ )

\begin{tabular}{lccccc}
\hline Variable & 1 & 2 & 3 & 4 & 5 \\
\hline 1. Attachment anxiety & - & $.43^{\star \star \star}$ & $.55^{\star \star \star}$ & $.54^{\star \star \star}$ & $.46^{\star \star \star}$ \\
2. Difficulties engaging with goals & - & - & $.44^{\star \star}$ & $.62^{\star \star \star}$ & $.42^{\star \star \star}$ \\
3. Impulse & - & - & - & $.72^{\star \star \star}$ & $.46^{\star \star \star}$ \\
4. Lack of strategies & - & - & - & $-48^{\star \star \star}$ \\
5. Paranoia & - & - & - & - \\
\hline
\end{tabular}

${ }^{\star \star \star} p<.001$.

Table 3. Correlation matrix for attachment avoidance and emotion regulation (Pearson's $r$ )

\begin{tabular}{lcccccc}
\hline Variable & 1 & 2 & 3 & 4 & 5 & 6 \\
\hline 1. Attachment avoidance & - & $.54^{\star \star \star}$ & $.39^{\star \star}$ & $.47^{\star \star \star}$ & $.69^{\star \star \star}$ & $.37^{\star \star}$ \\
2. Non-acceptance & - & - & $.34^{\star \star}$ & $.66^{\star \star \star}$ & $.39^{\star \star}$ & $.57^{\star \star \star}$ \\
3. Lack of awareness & - & - & - & $.54^{\star \star \star}$ & $.43^{\star \star \star}$ & $.27^{\star}$ \\
4. Lack of clarity & - & - & - & - & $-32^{\star}$ & $.38^{\star \star}$ \\
5. Suppression & - & - & - & - & - & $-32^{\star}$ \\
6. Paranoia & - & - & - & - & - \\
\hline
\end{tabular}

${ }^{\star} p<.05,{ }^{* *} p<.01,{ }^{\star * *} p<.001$.

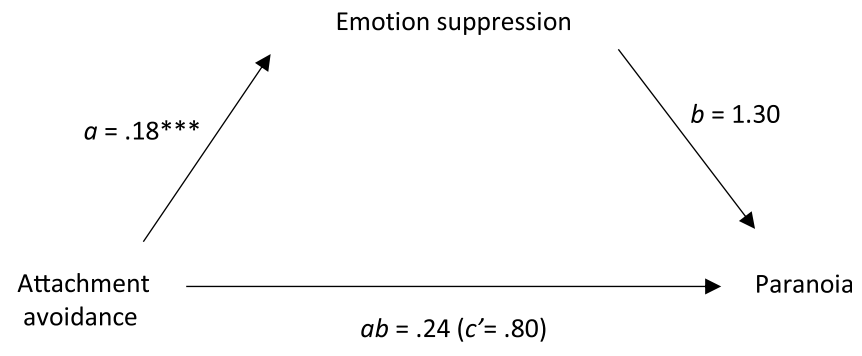

Figure 3. Mediation model testing if emotion suppression mediates the relationships between attachment avoidance and paranoia. Path coefficients are unstandardised regression coefficients. The value in parentheses is the direct effect $\left(c^{\prime}\right)$ of attachment avoidance on paranoia. ${ }^{\star} p<.05,{ }^{\star \star} p<.01,{ }^{\star \star \star} p<.001$.

The variance accounted for in paranoia increased to $38 \%$ in both models, when covariates were added.

\section{Exploratory analyses}

Table 2 gives the correlation coefficients between attachment anxiety, hyperactivating emotion regulation measures, and paranoia. In line with RQ1, there was a positive association between attachment anxiety and these strategies (difficulties engaging in goal-directed behaviours when upset, impulse control difficulties, and lack of access to emotion regulation strategies). These strategies also correlated with level of paranoia.

Table 3 gives the correlation coefficients between attachment avoidance, deactivating emotion regulation measures, and paranoia. In line with RQ2, there was a positive association between attachment avoidance and these strategies (emotional non-acceptance, lack of emotional awareness, lack of emotional clarity, and emotional suppression). These strategies also correlated with level of paranoia. 


\section{Discussion}

The positive associations between variables, and results of the mediation analyses, support theoretical assumptions about the role of emotion regulation in the relationship between attachment style and paranoia. Our results suggest that emotion dysregulation mediates the relationship between attachment anxiety and paranoia, and between attachment avoidance and paranoia in those with elevated levels of paranoia.

Level of paranoia in the current sample was comparable to clinical groups - in the moderatelysevere to severe ranges, and above the clinical cut-off for persecutory delusions, on average (see Table 1). Our results support theories which suggest that emotion dysregulation contributes to the maintenance of paranoia (Freeman and Garety, 2014; Freeman et al., 2002), and builds on these by showing that attachment style affects emotion dysregulation and therefore severity of paranoia (cf. Mikulincer and Shaver, 2012). These results are in line with previous research showing that attachment impacts emotion regulation (e.g. Owens et al., 2013) and paranoia (Murphy et al., 2020), and emotion regulation impacts paranoia (e.g. Lincoln et al., 2018). In addition, it contributes to the growing evidence that emotion regulation mediates this attachment and paranoia relationship (Ascone et al., 2020; Castilho et al., 2017; Jones, 2015; Udachina and Bentall, 2014) and addressed some of the limitations of this past research by using strong data analysis, controlling for confounders, and employing a priori analyses.

Our findings suggest that emotion regulation may be a valuable target in CBT for psychosis characterised by paranoia. Emotion regulation is not currently targeted in recommended CBT interventions (Morrison and Barratt, 2010), and attention to emotion regulation may improve the currently modest outcomes for CBT for psychosis (Laws et al., 2018). Indeed, preliminary research has shown that emotion regulation skills training is an effective intervention for reducing paranoia (Silva et al., 2020). This adds to the growing evidence that CBT interventions targeting specific maintenance factors associated with particular symptoms, such as paranoia, are likely to improve clinical outcomes for people with psychosis (e.g. Freeman et al., 2015).

Despite being correlated with both variables, emotion suppression did not mediate the relationship between attachment avoidance and paranoia. Previous research indicates that individuals with an avoidant attachment often use emotion suppression (Mikulincer and Shaver, 2019), and that emotion suppression increases paranoia (Nittel et al., 2018). This mediation model was significantly under-powered (Schoemann et al., 2017), which increases the likelihood of a Type II error. The lack of power in this model was partly due to the small relationship between the mediator (suppression) and dependent variable (paranoia), which conflicts with previous research finding a strong relationship between these variables (e.g. Nittel et al., 2018). It may also be that the diagnostic heterogeneity of the sample reduced the emotional suppression-paranoia relationship. Additionally, the suppression measure (ERQ) used in the current study consists of several negatively worded questions, which can cause participant confusion (Johnson et al., 2004; Patten, 1998).

Attachment avoidance correlated with deactivating emotion regulation strategies and attachment anxiety correlated with hyperactivating emotion regulation strategies. This is in line with previous research (Mikulincer and Shaver, 2007; Owens et al., 2013). These dysfunctional emotion regulation strategies correlated with paranoia, which again corroborates previous findings which suggest that dysfunctional emotion regulation increases level of paranoia (e.g. Westermann and Lincoln, 2011; Westermann et al., 2013). It may be that specific strategies are involved in mediating the relationship between the different attachment styles and paranoia - that hyperactivating emotion regulation mediates the relationship between attachment anxiety and paranoia, and deactivating emotion regulation mediates the relationship between attachment avoidance and paranoia. This now needs to be examined. 


\section{Limitations}

The results should be considered within the study's limitations. The study was cross-sectional, which limits the degree to which causal inferences can be made, although there is a strong theoretical basis for the temporal ordering of the variables (Fairchild and McDaniel, 2017). While relevant measured variables were controlled for (i.e. gender and recruitment pathway), there are likely to have been other potential confounding variables which were not measured, for example prescribed medication which can be used as an emotion regulation strategy. Such variables may have had an influence on observed effects.

While our sample had a reasonable balance of gender and wide age range, participants were majority White British (80.65\%), limiting the generalisability of the results. Furthermore, the sample was self-selecting and participants may not therefore be representative of the target population.

Mediation model 3 was significantly under-powered and used a questionnaire with negatively worded questions which may have caused confusion.

\section{Future research and clinical implications}

Future research should first examine the role of emotion regulation in the attachment-paranoia relationship using longitudinal or experimental designs, to determine causality. These studies should use a priori analyses to explore if hyperactivating emotion regulation strategies mediate the attachment anxiety-paranoia relationship, and if deactivating emotion regulation strategies mediate the attachment avoidance-paranoia relationship, as these have been explored post-hoc in the current study.

Following longitudinal/experimental studies, intervention studies are needed to explore the impact of emotion regulation skills training for individuals with paranoia, and specifically, whether those high in attachment anxiety benefit from emotion regulation skills training to reduce hyperactivating strategies (i.e. develop strategies to reduce emotional arousal and impulsivity), and whether those high in attachment avoidance benefit from emotion regulation skills training to reduce deactivating strategies (i.e. gain awareness and acceptance of emotions).

In terms of clinical implications, routine assessment of individuals' trait attachment style and emotion regulation strategies would help guide formulation and treatment planning in cognitive behavioural therapy for psychosis (cf. Berry et al., 2008; Gumley et al., 2014; Korver-Nieberg et al., 2014; Ludwig et al., 2019). People with attachment anxiety are likely to benefit from skills to reduce emotional arousal, whereas those with attachment avoidance are likely to benefit from skills to articulate and express emotional arousal.

\section{Conclusions}

Our study found that emotion regulation mediates the relationship between insecure attachment (anxiety/avoidance) and paranoia in a sample with clinical levels of paranoia. Our results show that hyperactivating emotion regulation is associated with attachment anxiety and paranoia, and deactivating emotion regulation is associated with attachment avoidance and paranoia. Research should now investigate causality and if attachment-based emotion regulation interventions are effective in reducing elevated levels of paranoia.

Acknowledgements. The authors would like to thank all who took part in the study, and the charities and clinicans for their support.

Financial support. This research received no specific grant from any funding agency, commercial or not-for-profit sectors.

Conflicts of interest. The authors declare none.

Ethics statements. Authors have abided by the Ethical Principles of Psychologists and Code of Conduct as set out by the BABCP and BPS. Ethical approval was granted by the University of Southampton Ethics Committee (ERGO ID: 53616; 
56538.A2), Health Research Authority (IRAS ID: 276512), Research Ethics Committee, and local NHS Trust Research and Development Department.

Data availability statement. The data that support the findings of this study are available from the corresponding author (O.P.), upon reasonable request.

Author contributions. Olivia Partridge: Conceptualization (equal), Data curation (lead), Formal analysis (lead), Investigation (equal), Methodology (equal), Project administration (equal), Resources (equal), Software (equal), Validation (equal), Visualization (equal), Writing - original draft (lead), Writing - review \& editing (equal). Tess Maguire: Conceptualization (equal), Investigation (equal), Methodology (equal), Project administration (equal), Resources (equal), Software (equal), Supervision (equal), Validation (equal), Visualization (equal), Writing - review \& editing (equal). Katherine NewmanTaylor: Conceptualization (equal), Investigation (equal), Methodology (equal), Project administration (equal), Resources (equal), Software (equal), Supervision (lead), Validation (equal), Visualization (equal), Writing review \& editing (lead).

\section{References}

Ascone, L., Schlier, B., Sundag, J., \& Lincoln, T. M. (2020). Pathways from insecure attachment dimensions to paranoia: the mediating role of hyperactivating emotion regulation versus blaming others. Psychology and Psychotherapy - Theory Research and Practice, 93, 72-87. https://doi.org/10.1111/papt.12208

Berry, K., Barrowclough, C., \& Wearden, A. (2008). Attachment theory: a framework for understanding symptoms and interpersonal relationships in psychosis. Behaviour Research and Therapy, 46, 1275-1282. https://doi.org/https://doi. org/10.1016/j.brat.2008.08.009

Berry, K., Bucci, S., \& Danquah, A. N. (2020). Attachment Theory and Psychosis: Current Perspectives and Future Directions. Routledge/Taylor \& Francis Group.

Berry, K., Wearden, A., Barrowclough, C., \& Liversidge, T. (2006). Attachment styles, interpersonal relationships and psychotic phenomena in a non-clinical student sample. Personality and Individual Differences, 41, 707-718. https://doi. org/10.1016/j.paid.2006.03.009

Bighelli, I., Salanti, G., Huhn, M., Schneider-Thoma, J., Krause, M., Reitmeir, C., Wallis, S., Schwermann, F., PitschelWalz, G., Barbui, C., Furukawa, T. A., \& Leucht, S. (2018). Psychological interventions to reduce positive symptoms in schizophrenia: systematic review and network meta-analysis. World Psychiatry, 17, 316-329. https://doi.org/10.1002/wps. 20577

Bowlby, J. (1969/1982). Attachment and Loss, Volume 1: Attachment. Basic Books.

Brennan, K. A., Clark, C. L., \& Shaver, P. R. (1998). Self-report measurement of adult attachment: an integrative overview. In Simpson, J. A., \& Rholes, W. S. (eds), Attachment Theory and Close Relationships (pp. 46-76). The Guilford Press.

Castilho, P., Martins, M. J., Pinto, A. M., Viegas, R., Carvalho, S., \& Madeira, N. (2017). Understanding the effect of attachment styles in paranoid ideation: the mediator role of experiential avoidance. Journal of Contextual Behavioral Science, 6, 42-46. https://doi.org/10.1016/j.jcbs.2016.11.007

Cooke, A. (2014). Understanding psychosis and schizophrenia. The British Psychological Society. https://www.bps.org.uk/ what-psychology/understanding-psychosis-and-schizophrenia

Dozier, M., \& Lee, S. W. (1995). Discrepancies between self- and other-report of psychiatric symptomatology: effects of dismissing attachment strategies. Development and Psychopathology, 7, 217-226. https://doi.org/10.1017/ S095457940000643X

Fairchild, A. J., \& McDaniel, H. L. (2017). Best (but oft-forgotten) practices: mediation analysis. American Journal of Clinical Nutrition, 105, 1259-1271. https://doi.org/10.3945/ajcn.117.152546

Fowler, J. C., Clapp, J. D., Madan, A., Allen, J. C., \& Oldham, J. M., \& Frueh, B. C. (2016). Emotion dysregulation as a cross-cutting target for inpatient psychiatric intervention. Journal of Affective Disorders, 206. doi: 10.1016/j.jad.2016.07.043.

Freeman, D. (2007). Suspicious minds: the psychology of persecutory delusions. Clinical Psychology Review, 27, $425-457$. https://doi.org/https://doi.org/10.1016/j.cpr.2006.10.004

Freeman, D. (2016). Persecutory delusions: a cognitive perspective on understanding and treatment. The Lancet Psychiatry, 3 , 685-692. https://doi.org/10.1016/S2215-0366(16)00066-3

Freeman, D., Dunn, G., Startup, H., Pugh, K., Cordwell, J., Mander, H., Černis, E., Wingham, G., Shirvell, K., \& Kingdon, D. (2015). Effects of cognitive behaviour therapy for worry on persecutory delusions in patients with psychosis (WIT): a parallel, single-blind, randomised controlled trial with a mediation analysis. The Lancet Psychiatry, 2, 305-313.

Freeman, D., \& Garety, P. (2014). Advances inunderstanding and treating persecutory delusions: A review. SocialPsychiatry and Psychiatric Epidemiology, 49, 1179-1189. https://doi.org/10.1007/s00127-014-0928-7

Freeman, D., Garety, P., Bebbington, P., Smith, B., Rollinson, R., Fowler, D., Kuipers, E., Ray, K., \& Dunn, G. (2005). Psychological investigation of the structure of paranoia in a non-clinical population. British Journal of Psychiatry: Journal of Mental Science, 186, 427-435. https://doi.org/10.1192/bjp.186.5.427 
Freeman, D., Garety, P. A., Kuipers, E., Fowler, D., \& Bebbington, P. E. (2002), A cognitive model of persecutory delusions. British Journal of Clinical Psychology, 41, 331-347. https://doi.org/10.1348/014466502760387461

Freeman, D., Loe, B. S., Kingdon, D., Startup, H., Molodynski, A., Rosebrock, L., Brown, P., Sheaves, B., Waite, F., \& Bird, J. C. (2019). The revised Green et al., Paranoid Thoughts Scale (R-GPTS): psychometric properties, severity ranges, and clinical cut-offs. Psychological Medicine, 1-10. doi: 10.1017/S0033291719003155

Gratz, K. L., \& Roemer, L. (2004). Multidimensional assessment of emotion regulation and dysregulation: development, factor structure, and initial validation of the difficulties in emotion regulation scale. Journal of Psychopathology and Behavioral Assessment, 26, 41-54. https://doi.org/10.1023/B:JOBA.0000007455.08539.94

Gross, J. J., \& John, O. P. (2003). Individual differences in two emotion regulation processes: implications for affect, relationships, and well-being. Journal of Personality and Social Psychology, 85, 348-362. https://doi.org/10.1037/00223514.85.2.348

Gumley, A. I., Taylor, H. E. F., Schwannauer, M., \& MacBeth, A. (2014). A systematic review of attachment and psychosis: measurement, construct validity and outcomes. Acta Psychiatrica Scandinavica, 129, 257-274.

Hayes, A. F. (2018). Introduction to Mediation, Moderation, and Conditional Process Analysis (2nd edn). Guilford Press.

Hugill, M., Fletcher, I., \& Berry, K. (2017). Investigation of associations between attachment, parenting and schizotypy during the postnatal period. Journal of Affective Disorders, 220, 86-94. https://doi.org/10.1016/j.jad.2017.05.040

Hutton, J., Ellett, L., \& Berry, K. (2017). Adult attachment and paranoia: an experimental investigation. Cognitive Behaviour Therapist, 10. https://doi.org/10.1017/S1754470X17000058

Johns, L. C., \& van Os, J. (2001). The continuity of psychotic experiences in the general population. Clinical Psychology Review, 21, 1125-1141. https://doi.org/https://doi.org/10.1016/S0272-7358(01)00103-9

Johnson, J. M., Bristow, D. N., \& Schneider, K. C. (2004). Did you not understand the question or not? An investigation of negatively worded questions in survey research. Journal of Applied Business Research, 20, 75-86. https://doi.org/10.19030/ jabr.v20i1.2197

Jones, J. E. (2015). The role of attachment, emotion regulation and recovery style in psychosis. Unpublished doctoral thesis, University of Birmingham.

Kimhy, D., Lister, A., Liu, Y., Vakhrusheva, J., Delespaul, P., Malaspina, D., Ospina, L. H., Mittal, V. A., Gross, J. J., \& Wang, Y. (2020). The impact of emotion awareness and regulation on psychotic symptoms during daily functioning. NPJ Schizophrenia, 6, 1-7. https://doi.org/10.1038/s41537-020-0096-6

Kimhy, D., Vakhrusheva, J., Jobson-Ahmed, L., Tarrier, N., Malaspina, D., \& Gross, J. J. (2012). Emotion awareness and regulation in individuals with schizophrenia: implications for social functioning. Psychiatry Research, 200, $193-201$.

Kline, R. B. (2015). Principles and Practice of Structural Equation Modeling. Guilford Publications.

Korver-Nieberg, N., Berry, K., Meijer, C. J., \& De Haan, L. (2014). Adult attachment and psychotic phenomenology in clinical and non-clinical samples: a systematic review. Psychology and Psychotherapy: Theory, Research and Practice, $87,127-154$.

Lavin, R., Bucci, S., Varese, F., \& Berry, K. (2020). The relationship between insecure attachment and paranoia in psychosis: a systematic literature review. British Journal of Clinical Psychology, 59, 39-65. https://doi.org/10.1111/bjc.12231

Laws, K. R., Darlington, N., Kondel, T. K., McKenna, P. J., \& Jauhar, S. (2018). Cognitive behavioural therapy for schizophrenia - outcomes for functioning, distress and quality of life: a meta-analysis. BMC Psychology, 6, 1-10. https://doi.org/10.1186/s40359-018-0243-2

Lincoln, T. M., Sundag, J., Schlier, B., \& Karow, A. (2018). The relevance of emotion regulation in explaining why social exclusion triggers paranoia in individuals at clinical high risk of psychosis. Schizophrenia Bulletin, 44, 757-767. https://doi. org/10.1093/schbul/sbx135

Ludwig, L., Werner, D., \& Lincoln, T. M. (2019). The relevance of cognitive emotion regulation to psychotic symptoms - a systematic review and meta-analysis. Clinical Psychology Review, 72, 101746.

Mikulincer, M., \& Shaver, P. R. (2007). Attachment in Adulthood: Structure, Dynamics, and Change. The Guilford Press.

Mikulincer, M., \& Shaver, P. R. (2012). An attachment perspective on psychopathology. World Psychiatry, 11, 11-15. https:// doi.org/https://doi.org/10.1016/j.wpsyc.2012.01.003

Mikulincer, M. \& Shaver, P. R. (2016). Attachment in Adulthood: Structure, Dynamics, and Change (2nd edn). The Guilford Press.

Mikulincer, M., \& Shaver, P. R. (2019). Attachment orientations and emotion regulation. Current Opinion in Psychology, 25, 6-10. https://doi.org/10.1016/j.copsyc.2018.02.006

Mikulincer, M., Shaver, P. R., \& Pereg, D. (2003). Attachment theory and affect regulation: the dynamics, development, and cognitive consequences of attachment-related strategies. Motivation and Emotion, 27, 77-102. https://doi.org/10.1023/A: 1024515519160

Morrison, A. P., \& Barratt, S. (2010). What are the components of CBT for psychosis? A Delphi study. Schizophrenia Bulletin, 36, 136-142. https://doi.org/10.1093/schbul/sbp118

Murphy, R., Goodall, K., \& Woodrow, A. (2020), The relationship between attachment insecurity and experiences on the paranoia continuum: a meta-analysis. British Journal of Clinical Psychology, 59, 290-318. https://doi.org/10.1111/bjc.12247 
National Institute for Health and Care Excellence (2014). Psychosis and Schizophrenia in Adults: Prevention and Management. https:/www.nice.org.uk/guidance/cg178/resources/psychosis-and-schizophrenia-in-adults-prevention-andmanagement-pdf-35109758952133

Nittel, C. M., Lincoln, T. M., Lamster, F., Leube, D., Rief, W., Kircher, T., \& Mehl, S. (2018). Expressive suppression is associated with state paranoia in psychosis: an experience sampling study on the association between adaptive and maladaptive emotion regulation strategies and paranoia. British Journal of Clinical Psychology, 57, 291-312. https://doi. org/10.1111/bjc.12174

Owens, K. A., Haddock, G., \& Berry, K. (2013). The role of the therapeutic alliance in the regulation of emotion in psychosis: an attachment perspective. Clinical Psychology \& Psychotherapy, 20, 523-530. https://doi.org/10.1002/cpp.1793

Patten, M. L. (1998). Questionnaire Research. Pyrczak Publishing.

Pickering, L., Simpson, J., \& Bentall, R. P. (2008). Insecure attachment predicts proneness to paranoia but not hallucinations. Personality and Individual Differences, 44, 1212-1224. https://doi.org/10.1016/j.paid.2007.11.016

Pilton, M., Bucci, S., McManus, J., Hayward, M., Emsley, R., \& Berry, K. (2016). Does insecure attachment mediate the relationship between trauma and voice-hearing in psychosis? Psychiatry Research, 246, 776-782. https://doi.org/10.1016/j. psychres.2016.10.050

Read, J., \& Gumley, A. (2008). Can attachment theory help explain the relationship between childhood adversity and psychosis? Attachment: New Directions in Psychotherapy and Relational Psychoanalysis, 2, 1-35.

Robson, G., \& Mason, O. (2015). Interpersonal processes and attachment in voice-hearers. Bevioural and Cognitive Psychotherapy, 43, 655-668. https://doi.org/10.1017/S1352465814000125

Schoemann, A. M., Boulton, A. J., \& Short, S. D. (2017). Determining power and sample size for simple and complex mediation models. Social Psychological and Personality Science, 8, 379-386.

Scott, M., Rossell, S. L., Meyer, D., Toh, W. L., \& Thomas, N. (2020). Childhood trauma, attachment and negative schemas in relation to negative auditory verbal hallucination (AVH) content. Psychiatry Research, 290. https://doi.org/10.1016/j. psychres.2020.112997

Silva, D., Maguire, T., McSherry, P., \& Newman-Taylor, K. (2020). Targeting affect leads to reduced paranoia in people with psychosis: a single case series. Behavioural and Cognitive Psychotherapy, 1-12. https://doi.org/10.1017/S1352465820000788

Sood, M., \& Newman-Taylor, K. (2020). Cognitive fusion mediates the impact of attachment imagery on paranoia and anxiety. Cognitive Therapy and Research. https://doi.org/10.1007/s10608-020-10127-y

Tabachnick, B. G., \& Fidell, L. S. (2013). Using Multivariate Statistics (6th edn). Pearson.

Udachina, A., \& Bentall, R. P. (2014). Developmental pathway to paranoia is mediated by negative self-concept and experiential avoidance. Psychosis - Psychological Social and Integrative Approaches, 6, 143-154. https://doi.org/10.1080/ 17522439.2013.810301

van der Gaag, M., Valmaggia, L. R., \& Smit, F. (2014). The effects of individually tailored formulation-based cognitive behavioural therapy in auditory hallucinations and delusions: a meta-analysis. Schizophrenia Research, 156, 30-37. https://doi.org/10.1016/j.schres.2014.03.016

Westermann, S., Boden, M. T., Gross, J. J., \& Lincoln, T. M. (2013). Maladaptive cognitive emotion regulation prospectively predicts subclinical paranoia. Cognitive Therapy and Research, 37, 881-885. https://doi.org/10.1007/s10608-013-9523-6

Westermann, S., \& Lincoln, T. M. (2011). Emotion regulation difficulties are relevant to persecutory ideation. Psychology and Psychotherapy: Theory, Research and Practice, 84, 273-287. https://doi.org/https://doi.org/10.1348/147608310X523019

Wickham, S., Sitko, K., \& Bentall, R. P. (2015). Insecure attachment is associated with paranoia but not hallucinations in psychotic patients: the mediating role of negative self-esteem. Psychological Medicine, 45, 1495-1507. https://doi.org/10. 1017/S0033291714002633

Cite this article: Partridge O, Maguire T, and Newman-Taylor K (2022). Pathways from insecure attachment to paranoia: the mediating role of emotion regulation. Behavioural and Cognitive Psychotherapy 50, 404-417. https://doi.org/10.1017/ S1352465822000029 